LIBERO GEROSA

Facoltà di Teologia di Lugano, Svizzera

\title{
DIRITTO UNIVERSALE E PARTICOLARE ALLA LUCE DELL'ERMENEUTICA CANONISTICA DI SAN GIOVANNI PAOLO II
}

Sommario: 1. Introduzione: le radici mistiche dell'attuazione culturale e strutturale della svolta ecclesiologica conciliare. - 2. La via innovativa e missionaria tracciata da Giovanni Paolo II alla canonistica del III millennio. - 2.1. Le principali novità dell'attività legislativa di Giovanni Paolo II. - 2.2. Le coordinate fissate da Giovanni Paolo II per lo sviluppo di una nuova ermeneutica canonistica. - 3. Il principio ecclesiologico della reciproca immanenza e la nuova definizione delle funzioni del diritto universale e del diritto particolare nella Communio Ecclesiarum. - 3.1. La communio Ecclesiarum in prospettiva ecclesiologico-canonistica. - 3.2. Le conseguene della communio Ecclesiarum a livello dei rapporti diritto universale-diritto particolare. - Conclusione.

\section{Introduzione: le radici mistiche dell'attuazione culturale e strutturale della svolta ecclesiologica conciliare}

«Non abbiate paura! Aprite, anzi spalancate le porte a Cristo» ${ }^{1}$ : tutti sono stati colpiti dalla forza di queste parole pronunciate il 22 ottobre 1978 da Giovanni Paolo II durante l'omelia della Santa Messa di inizio pontificato; molti hanno già allora intuito che con l'avvento del primo papa polacco sarebbero cambiate tante cose nel mondo

\footnotetext{
${ }^{1}$ Giovanni Paolo II, Omelia per l'inzio del pontificato, 22 Ottobre 1978, in: Insegnamenti di Giovanni Paolo II, Città del Vaticano 1978, vol. I, pp. 35-41, qui n. 5.
} 
intero $^{2}$; pochi però compresero che anche la Chiesa sarebbe andata incontro ad un profondo rinnovamento spirituale e strutturale. Eppure questo rinnovamento inizia fin da subito, nel gennaio 1979, quando Giovanni Paolo II - contro il parere dei suoi più stretti collaboratori ed alla decisione presa dal suo predecessore-compie il suo primo viaggio apostolico in Messico, partecipando alla riunione del CELAM di Puebla. Non si tratta di una decisione politica a favore della lotta senza quartiere contro il marxismo e le sue infiltrazioni nella teologia cattolica, bensì di una decisione pastorale capace di dimostrare concretamente come l'attuazione del Concilio Vaticano II passa dalla ricezione effettiva dell'ecclesiologia di comunione, che predilige la dimensione carismatica e missionaria di tutta la Chiesa ${ }^{3}$.

Tutti i successivi viaggi apostolici di Giovanni Paolo II rappresentano «un grande sostegno per le Chiese locali» ${ }^{4}$, ma è il primo viaggio in Messico ed il discorso di Puebla all'episcopato latino americano a segnare «l'inizio di una nuova storia per la Chiesa Cattolica», vissuta e promossa dal Papa Santo come "casa e scuola di comunione", come «famiglia» nella cui storia quotidiana si mette «armonicamente insieme unità e molteplicità, identità e diversità» ${ }^{5}$. Come emerge chiaramente nel discorso inaugurale del papa a Puebla, non si tratta solo di rilanciare la missione della Chiesa ovunque, ma soprattutto di prendere coscienza della svolta ecclesiologica epocale operata dal Concilio Vaticano II, perché «non c’è garanzia di un'azione evangelizzatrice seria e rigorosa, se manca un'ecclesiologia ben fondata. Innanzitutto perché evangelizzare è la missione essenziale, la vocazione propria, l'identità più profonda della Chiesa...(e) poi perché

\footnotetext{
${ }^{2}$ È il giudizio di A. Tornielli, L’ultimo miracolo. Perché Giovanni Paolo II è santo, Milano 2014, pp. 66-67.

${ }^{3}$ Cfr. LG 4,1 e 12,2 ed il commento in: L. Gerosa, Carisma e diritto nella Chiesa, Milano 1989, pp. 46-57.

${ }^{4}$ S. DzIwisz, Ho vissuto con un Santo. Conversazione con Gian Franco Svidercoschi, Milano 2013, p. 54.

${ }^{5}$ Ibid., p. 59.
} 
evangelizzare non è mai per nessuno un atto individuale e isolato, ma profondamente ecclesiale, un atto della Chiesa ${ }^{6}$.

Qual è la fonte originaria della chiarezza e della lucidità di tale giudizio? Da dove vengono la forza e la convinzione con cui il giovane papa polacco promuove una simile attuazione del Concilio Vaticano II, concretizzata poi negli anni successivi in un'attività legislativa davvero impressionante? Papa Benedetto XVI ha autorevolmente indicato la strada che conduce alla risposta giusta a tutte queste domande: «solo a partire dal suo rapporto con Dio è possibile capire anche il suo indefesso impegno pastorale» ${ }^{7}$ e con esso la sua capacità di interpretare ed attuare il Concilio Vaticano II. Giovanni Paolo II «era sempre in contemplazione e in ascolto di Dio» e proprio «nel ripetersi giorno per giorno, settimana dopo settimana, anno dopo anno, di questa profonda, autentica e totale immersione in Dio, sta il vero segreto di Giovanni Paolo II $»^{8}$.

È forse azzardato o poco scientifico per un canonista all'inizio del III millennio dell'era cristiana rifarsi all'esperienza mistica del legislatore ecclesiastico per spiegare le novità caratterizzanti l'attuale sistema giuridico della Chiesa Cattolica? Losservatore attento non può che rispondere di no! Infatti, se Papa Giovanni Paolo II ha saputo

\footnotetext{
${ }^{6}$ Giovanni Paolo II, Discorso inaugurale, in: Puebla: L'evangelizzazione nel presente e nel futuro dell'America Latina. Documenti, Bologna 1979, nr. 7: il testo definitivo si trova in: Insegnamenti di Giovanni Paolo II, Città del Vaticano 1979, vol. II/1, pp. 212-230, qui p. 218.

${ }^{7}$ Benedetto XVI, Mi è divenuto sempre più chiaro che Giovanni Paolo II fosse santo, in: Accanto a Giovanni Paolo II, a cura di W. Redzioch, Milano 2014, pp. 13-24, qui. p. 23.

${ }^{8}$ A.Tornielli, L'ultimo miracolo, op. cit., p. 146. L'autore parla di "preghiera infusa" (pp. 60-61), ma il termine esatto è "contemplazione infusa", ossia la forma più alta di contemplazione "perché assume ed eleva al massino grado la funzione contemplativa dello spirito" (Dizionario enciclopedico di spiritualità/I, a cura di E. Ancilli, Roma 1990, p. 620). Come tale, questa infusione segreta, pacifica ed amorosa di Dio che infiamma il credente contemplativo nello spirito di amore, è più volte descritta da San Giovanni della Croce (cfr. La mistica. Fenomenologia e riflessione teologica, a cura di E. Ancilli, Roma 1984, vol. I, pp. 572-574), a cui San Giovanni Paolo II si ispira fin da giovane.
} 
durante tutto il suo pontificato, anche e soprattutto essere legislatore universale della Chiesa Cattolica, «vedere oltre» ${ }^{9}$, aprire una nuova strada, assumendosi la responsabilità di «tracciare la direzione» ${ }^{10}$, ciò lo si deve soprattutto alla sua esperienza personale di fede, alla sua esperienza mistica di Dio. Ed oggi, più che mai, nessun teologo e nessun canonista deve aver paura di affermarlo, perché da tempo grandi teologi come Hans Urs von Balthasar, Yves Congar e Henri De Lubac, hanno non solo segnalato l'importanza teologica dell'autorità dei santi, ma anche denunciato fortemente come ogni disciplina teologica, diritto canonico compreso, se è privata del suo nesso intrinseco con l'esperienza mistica, la vita di fede, è destinata inevitabilmente a diventare sterile, priva di ogni «forza di attrazione» ${ }^{11}$. L'esperienza mistica di ogni fedele, di ogni credente, di ogni santo, ed in particolare di chi è investito di autorità nella Chiesa, costituisce un locus theologicus, un orizzonte ermeneutico per tutta la teologia e le sue singole discipline. Essa spinge la riflessione sistematica verso problemi ed orizzonti inesplorati, e lo fa quasi sempre con finalità pratiche ed immediate $^{12}$. Insomma, come il teologo, se nella sua ricerca scientifica prescinde completamente dal riferimento alla mistica, diventa un semplice "teorico di Dio"13, così il canonista che pretendesse di svolgere il suo lavoro scientifico a prescindere dalla teologia, ed in specie dall'ecclesiologia, diventerebbe muto, inefficace, perché dopo il Concilio Vaticano II il vecchio motto "canonista sine civilista, parum" deve essere mutato e completato con "canonista sine theologo,

\footnotetext{
${ }^{9}$ Ibid., p. 163.

${ }^{10}$ S. Dziwisz, Ho vissuto con un santo, op. cit., p. 143.

${ }^{11}$ G. STRZELCZy K, L'esperienza mistica come locus theologicus, Lugano 2005, p. 54, il giudizio si fonda su teologi autorevolissimi: H. U. VON BAlthasar, Teologia e santità, in: Verbum caro, Brescia 19854, pp. 200-229; Y. Congar, La fede e la teologia, Roma 1967, p. 207; ma anche: R. Fisichella, Il Magistero teologico di Giovanni Paolo II. Saggio introduttivo, in: Giovanni Paolo II, Tutte le Encicliche. Testo latino a fronte, a cura di R. Fisichella, Città del Vaticano 2010, pp. 7-76, qui p. 12.

${ }^{12}$ G. Strzelczy K, L'esperienza mistica come locus theologicus, op. cit., p. 101.

${ }^{13}$ È il giudizio pungente di H. De Lubac, Mistica e mistero cristiano, Milano 1979, pp. 3-38, qui p. 21.
} 
nihil"14. In altri termini, dimenticando e addirittura censurando la svolta ecclesiologica conciliare, sviluppata attorno alla categoria teologica di communio, che costituisce il principio formale di tutto il diritto canonico post-conciliare ${ }^{15}$, il canonista cesserebbe di "sentire cum Ecclesia"16 e quindi di interpretare ed applicare correttamente le norme codiciali, ossia secondo lo spirito innovativo e missionario di chi le ha promulgate: il mistico e santo Papa, Giovanni Paolo II.

\section{La via innovativa e missionaria tracciata da Giovanni Paolo II alla canonistica del III millennio}

2.1. Le principali novità dell'attività legislativa di Giovanni Paolo II

Sono per lo meno sei; eccole.

- Innanzitutto Giovanni Paolo II è il primo Papa della storia a promulgare due codici di diritto canonico, uno per la Chiesa Cattolica Latina ed uno per le 21 Chiese Orientali, convinto com'è che un vero cattolico «deve avere due polmoni, cioè quello orientale e quello occidentale» ${ }^{17}$.

- In secondo luogo, Papa Giovanni Paolo II non ha neppure teoricamente escluso la possibilità che all'interno della cosiddetta communio Ecclesiarum sia possibile arrivare un giorno alla promulgazione di diversi insiemi di leggi canoniche particolari per i singoli continenti, vista l'intuizione profetica con cui ha chiamato a riflettere

\footnotetext{
${ }^{14}$ Il motto antico è così interpretato da: J.L. Gutierrez, Alcune questioni sull'interpretazione della legge, Apollinaris 60(1987), pp. 507-525, qui p. 520.

${ }^{15}$ Cfr. OT 16,4 ed il prezioso commento di: H. MüLleR, Utrum "communio" sit principium formale-canonicum nunc codificationis Iuris Canonici Ecclesiae Latinae?, Per RMCL 74(1985), pp. 85-108.

${ }^{16}$ Lo segnalava ben prima del Concilio Vaticano II un grande canonista laico italiano: V. Del GiUdice, Istituzioni di diritto canonico, Milano 1936, p. 6.

${ }^{17}$ Nei suoi viaggi apostolici e nei suoi scritti, Papa Giovanni Paolo II parla spesso di un'Europa che respira con «due polmoni», cfr. A. RICCARDI, Giovanni Paolo II. La biografia, Cinisello Balsamo (Milano), 2011, p. 160.
} 
sull'evangelizzazione i loro Vescovi in Sinodi specifici non solo per l'Europa, ma anche per gli altri continenti ${ }^{18}$.

- In terzo luogo non può essere sottaciuto che il Codice di Diritto Canonico, promulgato da Papa Giovanni Paolo II il 25 gennaio 1983 ed unanimalmente riconosciuto come «vera pietra miliare» sulla via da lui aperta per il completo rinnovamento del diritto della Chiesa Cattolica, è considerato da un suo stretto collaboratore, il Presidente emerito del Pontificio Consiglio per i testi legislativi Julian Card. Herranz non come un punto d'arrivo, bensì e soprattutto come «un punto di partenza» ${ }^{19}$. E difatti l'elenco dei documenti o testi legislativi che hanno via via configurato nei primi 25 anni lo sviluppo normativo postcodiciale è lunghissimo e fra essi spiccano per importanza il già citato Codice dei Canoni delle Chiese Orientali, promulgato da Giovanni Paolo II nel 1990, e la Costituzione apostolica sulla Curia Romana, pubblicata dallo stesso Pontefice nel $1988^{20}$. Quest'ultima, la cosiddetta "Pastor bonus», assieme al CIC e al CCEO costituiscono un vero e proprio nuovo "Corpus Iuris Canonici», che - come affermato dallo stesso Pontefice Giovanni Paolo $\mathrm{II}^{21}$ - va considerato

${ }^{18}$ A tale riguardo cfr. A. RicCARd, Giovanni Paolo II. La biografia, op. cit., pp. 433-434, ma anche E. Corecco, Prospettive per la "Lex Ecclesiae fundamentalis " e la revisione del diritto canonico nel documento di Puebla, Il diritto ecclesiastico, gennaio-marzo (1980), pp. 1-23; L. Gerosa, L'interpretazione della legge nella Chiesa. Principi, paradigmi, prospettive, Lugano 2001, soprattutto pp. 47-61.

${ }^{19}$ J. Herranz, Il Codice di Diritto canonico e il successivo sviluppo normativo, in: La legge canonica nella vita della Chiesa. Indagine e prospettive nel segno del recente magistero pontificio. Atti del Convegno di studio tenutosi nel XXV anniversario della promulgazione del Codice di Diritto Canonico, Città del Vaticano 2008, pp. 47-59, qui p. 50 e p. 48.

${ }^{20}$ Il testo ufficiale della CA «Pastor bonus» si trova in: AAS 80(1988), pp. 841-934 e quello della CA «Sacri canones» con cui viene promulgato il CCEO in: AAS 82(1990), pp. 1038-1039.

${ }^{21}$ Cfr. Giovanni Paolo II, CA «Sacrae disciplinae leges» (AAS 75, 1983), pars II, XI; id., «Pastor bonus», Nr. 2; id., Discorso al «Common Law Society of Great Britain and Ireland» (22 maggio 1992), Communicationes 24(1992), p. 10 ed il giudizio unanime di: J. Herranz, Il Codice di Diritto canonico e il successivo sviluppo normativo, op. cit., pp. 53-55 e P. ERDö, Rigidità ed elasticità delle strutture normative nel dialogo 
come strumento "corrispondente in pieno alla natura della Chiesa» e perciò come «strumento di comunione» al «servizio di un più profondo mistero di grazia».

- In quarto luogo, come se non bastasse, benché la fecondità innovativa di un legislatore non abbia nulla o poco da spartire con l'inflazione o il proliferare delle leggi da lui promulgate, Papa Giovanni Paolo II a scanso di equivoci e quasi per voler confermare «l'intrinseca vitalità e dinamicità dello stesso corpo normativo» ${ }^{22}$, da lui continuamente aggiornato, integrato e perfezionato, non manca di precisare «expressis verbis» che «in realtà, l'interpretazione autentica della Parola di Dio, operata dal Magistero della Chiesa, ha valore giuridico nella misura in cui riguarda l'ambito giuridico, senza aver bisogno di nessun ulteriore passaggio formale per diventare giuridicamente e moralmente vincolante ${ }^{23}$. In altri termini, non solo le leggi canoniche promulgate formalmente come tali, ma anche altri interventi del Magistero pontificio di natura prevalentemente pastorale o magisteriale possono avere una valenza giuridica non trascurabile, che concorre assieme alle leggi canoniche vere e proprie, decreti generali ed istruzioni, a comporre quell'insieme normativo o «Corpus Iuris Canonici», capace di tracciare la «rotta della barca di Pietro» ${ }^{24}$.

- In quinto luogo va ricordato che all'innegabile imponente attività legislativa di Giovanni Paolo II corrisponde un altrettanto grande ed innovativo sforzo di aggiornamento e completamento delle leggi canoniche codificate. Infatti, sia sotto il profilo della sistematica giuridica sia sotto quello materiale dei suoi principali contenuti normativi, è fuori di dubbio che il CIC abbia recepito - per lo meno nella sua sostanza - la lezione conciliare della Chiesa come communio. Ad

ecumenico, in: La legge canonica nella vita della Chiesa, op. cit., pp. 149-178, qui p. 167.

${ }^{22}$ Cfr. J. Herranz, Il Codice di Diritto canonico e il successivo sviluppo normativo, op. cit., p. 56.

${ }^{23}$ Giovanni Paolo II, Discorso alla Rota Romana, 29 gennaio 2005, AAS 97(2005), p. 166.

${ }^{24}$ J. Herranz, Il Codice di Diritto canonico e il successivo sviluppo normativo, op. cit., p. 53. 
esempio, nei suoi libri centrali (II, III e IV), da una parte sono messi in risalto proprio quegli elementi segnalati da Giovanni Paolo II come più significativi sulla Costituzione Apostolica Sacrae Disciplinae Leges, ossia: la categoria di Popolo di Dio per definire la struttura costituzionale della Chiesa, quella di servizio per cogliere la funzione della gerarchia, quella di communio per stabilire la partecipazione di tutti i fedeli alla missione della Chiesa ed all'esercizio dei tria munera Christi, nonché la collocazione giuridica del Christifidelis ed in particolare dei laici; dall'altra, sempre in questi libri centrali del nuovo Codice, per la prima volta Parola e Sacramento appaiono come elementi portanti di tutta la struttura ecclesiale ${ }^{25}$.

- Infine, rispetto al Codice del 1917, quello nuovo del 1983 presenta due novità così importanti da giustificare, almeno in parte $\mathrm{e}$ post factum, la sua realizzazione, nonostante le resistenze di coloro che, durante il lavoro di riforma, hanno sostenuto la necessità che la Chiesa Cattolica optasse per una decodificazione delle sue leggi. La prima novità consiste nel fatto che la nuova codificazione delle leggi canoniche non è più guidata in primo luogo dalla ricerca di una formulazione e sistemazione razionale delle norme, bensì dall'esigenza di svilupparle secondo i loro nessi con i contenuti della fede. Pertanto ultimamente parlando il principio della certezza teologica prevale su quello della certezza giuridica. La seconda novità è data dal cambiamento d'identità del soggetto principale di tutta la struttura giuridico-ecclesiale: non più il chierico, ma il Christifidelis, cioè il fedele, ossia il semplice battezzato è la figura primordiale soggiacente a quella di laico, chierico e religioso e come tale soggetto di diritti e doveri nella Chiesa mistero di comunione.

\footnotetext{
${ }^{25}$ Per una valutazione dettagliata della sistematica del nuovo Codice, $\mathrm{cfr}$. H. Schmitz, De ordinatione systematica novi Codicis Iuris Canonici recogniti, Periodica 68(1979), pp. 171-200. Sugli aspetti materiali e sui problemi posti dal fatto di procedere ad una nuova codificazione delle leggi della Chiesa, cfr. E. Corecco, Presupposti culturali ed ecclesiologici del nuovo Codex, in: Il nuovo Codice di Diritto Canonico. Aspetti fondamentali della codificazione postconciliare, a cura di S. Ferrari, Bologna 1983, pp. 37-68.
} 
Tutte queste novità, ed in particolare le ultime due, hanno un significato decisivo anche a livello dell'interpretazione e dell'applicazione del diritto canonico vigente. E proprio a livello dell'interpretazione ed applicazione delle leggi canoniche si esprime maggiormente la genialità di Giovanni Paolo II.

2.2. Le coordinate fissate da Giovanni Paolo II per lo sviluppo di una nuova ermeneutica canonistica

Se durante la riforma è prevalso lo sforzo di ricercare in profondità le ragioni teologiche e fondative delle norme canoniche, subito dopo la promulgazione del CIC nel 1983 l'interesse dei canonisti si è spostato sui problemi dell'interpretazione e dell'applicazione delle leggi nella Chiesa ${ }^{26}$. Con l'immediata istituzione del Pontificio Consiglio per l'interpretazione dei testi legislativi, Giovanni Paolo II li anticipa non solo cronologicamente. Infatti, con questa iniziativa, da grande comunicatore com'era, dimostra apertamente due cose. Innanzitutto di essere cosciente che per la Chiesa avere una solida e fondata tecnica interpretativa è fondamentale, perché ogni messaggio, anche giuridico, «acquista vera consistenza nella comunicazione agli interessati $»^{27}$. In secondo luogo di volere accanto a sé un dicastero, capace di "promuovere la disciplina comune a tutta la Chiesa", analogamente a quanto svolto dalla Congregazione per la Dottrina della fede in ordine al dovere e alla volontà di «promuovere e difendere l'unità della fede» ${ }^{28}$.

Questa primaria fondazione ecclesiologica, data da Giovanni Paolo II al nuovo dicastero, da una parte evidenzia come la funzione di quest'ultimo non si limita alla fase puramente tecnica dell'interpretazione vera e propria, ma si estende anche alla fase

\footnotetext{
${ }^{26}$ Cfr. J. Herranz Casado, L'interpretazione autentica: il Pontificio Consiglio per l'interpretazione dei testi legislativi, in: AA.VV., Il diritto della Chiesa. Interpretazione e prassi, Città del Vaticano 1996, pp. 65-93, qui p. 65.

${ }^{27}$ V. Frosini, Lezioni di teoria dell'interpretazione giuridica, Milano 1989, p. 108.

${ }^{28}$ Questo illuminante parallelismo è proposto dall'allora Presidente del Pontificio Consiglio per l'interpretazione dei testi legislativi sulla base dell'insegnamento di LG 23; cfr. J. Herranz CASAdo, L'interpretazione autentica, op. cit., p. 66.
} 
formativa del complesso sistema canonico ${ }^{29}$. Dall'altra parte, dato che il compito principale di questo nuovo dicastero è la cosiddetta interpretazione autentica per modus $\operatorname{legis}^{30}$, forma peculiare dell'interpretazione di testi legislativi che non trova riscontro adeguato negli ordinamenti civili ${ }^{31}$, con la sua istituzione, Giovanni Paolo II dimostra di essere lucidamente consapevole della specificità propria del diritto della Chiesa e della necessità di favorire attraverso una interpretazione della legge canonica un effettivo miglioramento sia nella tecnica legislativa, sia nell'attuazione della «communio in regimine». E con ciò Giovanni Paolo II da una parte valorizza la tradizione canonica che vede nella «interpretatio» una "aperta significatio legis» ${ }^{32}$, dall'altra la rivitalizza ponendo nuove basi all'ermeneutica canonistica nel suo insieme.

Il parallelismo fra la funzione del Pontificio Consiglio per l'interpretazione dei testi legislativi e quella della Congregazione per la Dottrina della Fede ha radici profonde, che vanno ricercate nel costante interagire fra teologia dogmatica e diritto canonico. Infatti, come è stato messo in luce già nel lontano 1980 a Friburgo da Eugenio

\footnotetext{
${ }^{29}$ Dall'Annuario Pontificio 2000 in poi la denominazione del nuovo dicastero «Pontificio Consiglio per i testi legislativi » non fa più riferimento all'interpretazione, ma la sostanza della sua funzione e delle sue competenze rimane immutata, cfr. in merito G. InCitTi, L'interpretazione e il Pontificio Consiglio per (l'interpretazione dei) $i$ testi legislativi, in: Associazione Canonistica Italiana, Fondazione del diritto. Tipologia e interpretazione della nuova canonistica, Milano 2001, pp. 153-178, qui p. 155.

${ }^{30}$ Sulle diverse categorie di interpretazione cfr. L. MüLLER, Authentische Interpretation - Auslegung kirchlicher Gesetze oder Rechtsfortbildung?, AfkKR 164(1995), pp. 353-375 e L. Gerosa, L'interpretazione della legge nella Chiesa. Principi, paradigmi, prospettive, Lugano 2001.

${ }^{31}$ A tale riguardo, cfr. J. HerRanz CASAdo, L'interpretazione autentica, op. cit., p. 68 e R.J. CASTIllo LaRA, De iuris canonici autentica interpretazione in actuositate Pontificae Cammissionis ademplenda, Communicationes 20(1988), p. 267.

${ }^{32}$ F. SuArez, De legibus, Lib. VI: De interpretazione, mutatione et cessatione legis, in: Opera Omnia, Paris 1856, vol. V-VI, qui ripreso da J. Herranz Casado, L'interpretazione autentica, op. cit., p. 67.
} 
Corecco ${ }^{33}$, alle quattro tappe principali di sviluppo della teologia dogmatica corrispondono altrettante tappe di sviluppo del diritto canonico. L'indicazione metodologica data dal Concilio Vaticano II permette di stabilire perciò un interessante parallelismo fra il rinnovamento epistemologico conosciuto dalla canonistica post-conciliare ed il carattere preminentemente sapienziale del diritto canonico del primo millennio, senza nulla togliere ai nuovi fondamenti ecclesiologici messi in luce dall'insegnamento conciliare sulla Chiesa come comunione $\mathrm{e}$ al ritrovato interesse per la centralità dell'antropologia da parte della scienza del diritto ecclesiale. In tutto ciò non è certamente estraneo l'influsso esercitato dall'azione di Giovanni Paolo II a livello del governare, legiferare ed interpretare durante tutto il suo lungo e fecondo pontificato.

Anzi, il fatto che la Chiesa Cattolica, quale communio Ecclesiarum abbia ormai due codici di diritto canonico è un «novum» assoluto che evidenzia come il pluralismo giuridico sia un dato di fatto innegabile e riconosciuto dalla Suprema Autorità ecclesiastica; i principi che hanno guidato questo sforzo epocale di codificazione del proprio diritto da parte della Chiesa Cattolica, principi fissati con estrema chiarezza dalla costituzione apostolica di promulgazione del primo dei due codici, sono evidentemente imprescindibili per una corretta interpretazione delle leggi canoniche in vigore.

Ma qual è l'essenza di questo sforzo? È ancora Giovanni Paolo II a precisarlo: innanzitutto questo duplice lavoro di codificazione "potrebbe intendersi come uno sforzo di tradurre il linguaggio canonistico... l'ecclesiologia conciliare» ${ }^{34}$; in secondo luogo per rispondere correttamente alla questione circa la natura stessa di entrambi i codici di diritto canonico «... bisogna riandare con la mente al lontano

\footnotetext{
${ }^{33}$ A tale riguardo cfr. E. Conecco, Considerazioni sul problema dei diritti fondamentali del cristiano nella Chiesa e nella Società. Aspetti metodologici della questione, in: Id., Ius et communio. Scritti di Diritto canonico, a cura di G. Borgonovo - A. Cattaneo con Prefazione di A. Scola, Lugano 1997, I. Vol., pp. 245-278, qui soprattutto pp. 246-253.

${ }^{34}$ Giovanni Paolo II, Sacrae disciplinae leges, cpv 18.
} 
patrimonio di diritto contenuto nei libri del Vecchio e Nuovo Testamento dal quale, come dalla sua prima sorgente, proviene tutta la tradizione giuridico-legislativa della Chiesa...» ${ }^{35}$.

Alla luce di tali considerazione, appare abbastanza chiaro che lo scopo della duplice codificazione canonica «... non può in nessun caso essere quello di sostituire la fede, la grazia, i carismi e soprattutto l'amore nella vita della Chiesa e dei fedeli. Al contrario il Codice mira piuttosto a dare alla società ecclesiale un ordine che dia all'amore, alla grazia e ai carismi il primato e contemporaneamente faciliti il loro organico sviluppo nella vita della società ecclesiale, come pure delle singole persone che ad essa appartengono. Il codice, dal momento che è il documento legislativo primario della Chiesa, fondato sull'eredità giuridica e legislativa della Rivelazione della Tradizione, è da considerare come uno strumento indispensabile, grazie al quale si garantisce l'ordine necessario alla vita personale e sociale, come pure all'attività della Chiesa stessa» ${ }^{36}$.

Rivelazione, Tradizione e Codice costituiscono dunque i fondamenti ineliminabili che concorrono a definire la struttura complessa e specifica della Chiesa.

Papa Giovanni Paolo II ha illustrato questo «carattere di complementarietà» ${ }^{37}$ del diritto canonico codificato durante la presentazione ufficiale del CIC il 3 febbraio 1983, usando l'immagine di un triangolo che si pone - riguardo all'applicazione e all'interpretazione del diritto canonico, a motivo dello stretto nesso fra vita di fede e ordinamento giuridico ecclesiale - come nuovo principio metodologico ed ermeneutico da seguire incondizionatamente: «accanto al libro che contiene gli atti conciliari c'è ora il nuovo codice ecclesiastico, e questa mi pare un'accoppiata giustamente efficace e significativa... In

\footnotetext{
${ }^{35}$ Ibid., cpv 13.

${ }^{36}$ Ibid., cpv 16.

${ }^{37}$ Ibid., cpv 20; Su come i documenti del Concilio Vaticano II siano da considerare quale «contesto» delle norme codiciali, cfr. L. MülLER, Die Lehre des zweiten Vatikanischem Konzils als Kontext zum Interpretation kirchenrechtiler Normen, AfkKR 169(2000), pp. 469-481.
} 
chiusura vorrei mostrarvi come indicazione e memoria, una sorta di triangolo ideale: in alto la Sacra Scrittura, da una parte i documenti del Vaticano II, dall'altra il nuovo Codice di Diritto canonico. E per salire in modo ordinato e coerente da questi due libri della Chiesa del $\mathrm{XX}$ secolo a quella vetta più alta e immutabile, si dovranno percorrere i lati di questo triangolo senza negligenze e omissioni e prestando attenzione alle necessarie relazioni: il magistero tutto - potrei dire dei passati concili ecumenici e anche (naturalmente tralasciando le norme transitorie e pregresse) quella eredità di sapienza giuridica che appartiene alla Chiesa» ${ }^{38}$.

Tale indirizzo metodologico può essere oggi così graficamente schematizzato e completato:

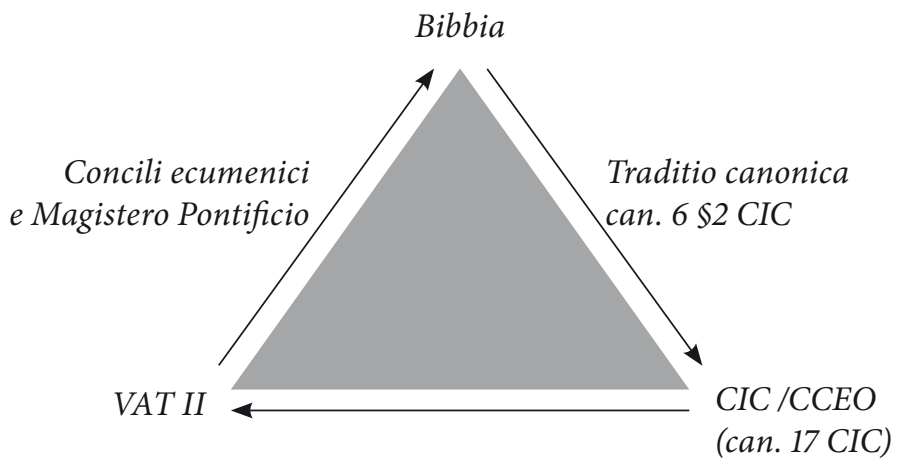

Questo triangolo, che costituisce ormai la base solida ed imprescindibile per una corretta ermeneutica canonistica, proprio nei suoi aspetti apparentemente più innovativi si ricollega armoniosamente e senza alcuna meccanicità alla migliore dottrina canonistica sull'interpretazione delle leggi. Infatti, il riconoscimento che la «lex canonica» abbia per sua natura bisogno di una «declaratio

\footnotetext{
${ }^{38}$ Giovanni Paolo II, Discorso del 3 febbraio 1983, AAS 75(1983), pp. 455-465, qui p. 463; cfr. anche H. Schmitz, Wertungen des Codex Iuris Canonici. Versuch einer ersten Bilanz, AfkKR 154(1985), pp. 19-57.
} 
doctrinalis», o di una interpretazione, fa parte di tutta la storia del diritto canonico ${ }^{39}$.

\section{Il principio ecclesiologico della reciproca immanenza e la nuova definizione delle funzioni del diritto universale e del diritto particolare nella communio Ecclesiarum}

Se all'alba del III millennio, all'interno del suo grande sforzo di concepire e promuovere l'aggiornamento conciliare come qualche cosa che viene dal profondo della Chiesa e non da un superficiale adattamento ai gusti della società contemporanea, Giovanni Paolo II riesce - come si è appena visto - a porre le basi solide per un nuovo linguaggio, pastorale e giuridico ${ }^{40}$, grazie al quale ricentrare tutto in Cristo, persino la riforma della struttura giuridica della Chiesa, che lui stesso definisce "casa e scuola della comunione», proprio nella lettera apostolica "Novo millennio ineunte» ${ }^{41}$ con cui indice l'Anno giubilare 2000, ciò è dovuto in gran parte - come è stato autorevolmente e recentemente testimoniato dal Cardinale Stanislao Dziwisz - a quel "progetto di Chiesa" che Karol Wojtyla aveva maturato negli anni polacchi, alla scuola del Concilio Vaticano II. E che, una volta diventato Papa, trovò la sua realizzazione sul piano dell'universalità, in particolar modo grazie alle prime tre encicliche, il cosiddetto trittico trinitario. Progetto che era segnato da una ecclesiologia fortemente cristocentrica: la Chiesa può considerarsi fine a se stessa, ma ha la sua ragion d'essere solo ed esclusivamente in Cristo. E da qui, poi,

\footnotetext{
${ }^{39}$ È l'autorevole giudizio di: B. E. Ferme, I principi interpretativi e l'inventiva interpretativa, Periodica 87(1998), pp. 191-213, qui p. 191 e p. 192; a tale riguardo cfr. pure: P. Grossi, L'ordine giuridico medievale, Roma-Bari 2003, pp. 162-168 e L. GerosA, La nuova ermeneutica canonistica di Giovanni Paolo II. Introduzione al Convegno internazionale di Lugano, in: Giovanni Paolo II: legislatore della Chiesa. Fondamenti, innovazioni e aperture (Atti del Convegno di studio, Lugano 22-23 marzo 2012), a cura di L. Gerosa, Città del Vaticano 2013, pp. 35-48, soprattutto pp. 43-45.

${ }^{40} \mathrm{Su}$ come Giovanni Paolo II abbia cercato in tutti i campi della vita ecclesiale «un nuovo linguaggio per dire e vivere quanto credeva», cfr. A. RICCARDI, Giovanni Paolo II. La biografia, op. cit., p. 255 e p. 512.

${ }^{41}$ In AAS 93(2001), pp. 266-300.
} 
discendeva la centralità della persona umana. Dunque: non più una Chiesa dalla struttura verticistica, monolitica, istituzionalizzata, ma una Chiesa che è "casa e scuola di comunione", una Chiesa famiglia, e che, incarnandosi a fondo nella storia quotidiana, mette armonicamente insieme unità e molteplicità, identità e diversità ${ }^{42}$.

Questo "progetto di Chiesa", profondamente conciliare ed abbozzato espressamente per la prima volta da Giovanni Paolo II nello storico discorso di Puebla, ha il suo perno strutturale nel principio ecclesiologico dell'immanenza reciproca fra universale e particolare nella Chiesa come communio Ecclesiarum. Vale la pena di approfondirlo brevemente alla luce della nuova ermeneutica canonistica per poter trarre tutte le conseguenze pastorali e missionarie, auspicate dal Santo Papa Giovanni Paolo II.

\subsection{La communio Ecclesiarum in prospettiva ecclesiologico-canonistica}

Anche se l'espressione communio Ecclesiarum ricorre una volta sola nei testi conciliari ${ }^{43}$, il concetto della communio Ecclesiarum rappresenta - per lo meno a livello giuridico-costituzionale - la chiave di volta di tutta l'ecclesiologia del Concilio Vaticano $\mathrm{II}^{44}$. Fra i commentatori

\footnotetext{
${ }^{42}$ S. Dziwisz, Ho vissuto con un santo, op. cit., pp. 58-59.

${ }^{43}$ Cfr. AG 19,3.

${ }^{44}$ Sulla centralità del termine di communio in tutta l'ecclesiologia del Concilio Vaticano II, cfr. W. Aymans, Die Communio Ecclesiarum als Gestaltgesetz der einen Kirche, in: ID., Kirchenrechtliche Beiträge zur Ekklesiologie, Berlin 1995, pp. 17-39; O. SAIER, "Communio» in der Lehre des Zweiten Vatikanischen Konzils. Eine rechtsbegriffliche Untersuchung, München 1973; W. KASPER, Zukunft aus der Kraft des Konzils. Die ausserordentliche Bischofssynode '85. Die Dokumente mit einem Kommentar, Freiburg-Basel-Wien 1986, pp. 33-40 e pp 88-97. Per un'analisi dei diversi significati ecclesiologici del principio della communio Ecclesiarum cfr. soprattutto: J. Ratzinger, Das neue Volk Gottes, Düsseldorf 1969, pp. 205 ss.; J. KoMONCHAK, Die Kirche ist universal als Gemeinschaft von Ortskirchen, Concilium 17(1981), pp. 471-476; M. KeHL, Ecclesia universalis. Zur Frage nach dem Subjekt der Universalkirche, in: E. Klinger - K. Wittstadt (hrsg. von), Glaube im Prozess (FS K. RAHNER), Freiburg 1984, pp. 240-257; G. GRESHAKE, «Zwischeninstanzen» zwischen Papst und Ortsbischöfen. Notwendige Voraussetzung für die Verwirklichung
} 
non manca chi vede nella definizione della Chiesa come «comunione di Chiese» una vera e propria «svolta copernicana» ${ }^{45}$. Comunque, al di là di ogni enfasi è chiaro che l'essenza strutturale del mistero della Chiesa come communio Ecclesiarum è quella della piena immanenza reciproca della Chiesa universale nelle e dalle Chiese particolari, colta dalla formula in quibus et ex quibus di LG 23,1 e sviluppata in altri testi paralleli ${ }^{46}$.

Tre sono i principali significati di questa formula sintetica:

a) la Chiesa universale non esiste in se stessa, quasi possedesse una consistenza e un luogo di insediamento (ad esempio Roma) propri, ma esiste e si concretizza laddove essa si realizza in una (all'inizio in Gerusalemme) o più Chiese particolari (come nei secoli successivi);

b) la Chiesa universale è formata da tutte le Chiese particolari e perciò non è una realtà astratta, ma storicamente concreta, che coincide di fatto con tutte le Chiese particolari esistenti;

c) in ogni Chiesa particolare sono ontologicamente presenti tutte le altre Chiese particolari, attraverso la mediazione della Chiesa universale, da esse costituita ${ }^{47}$.

der Kirche als "communion Ecclesiarum», in: H. Müller - H. J. Pottmeyer (hrsg. von), Die Bischofskonferenz. Theologischer und juristischer Status, Düsseldorf 1989, pp. 88-115; H. J. Ротtмeyer, Kirche als communio, Stimmen der Zeit 210(1992) 579-589; M. KeHL, Die Kirche. Eine katholische Ekklesiologie, Würzburg 1992, pp. 366-384; ID., Wohin geht die Kirche? Eine Zeitdiagnose, Freiburg-Basel-Wien 1996, pp. 80-98. Un'esposizione riassuntiva e completa della disputa si trova anche nella dissertazione: M. Werneke, Ius universale-Ius Particulare. Zum Verhältnis von Universal und Partikularrecht in der Rechtsordnung der lateinischen Kirche unter besonderer Berücksichtigung des Vermögensrechts, Paderborn 1998, pp. 17-81.

${ }^{45}$ P. Neuner, Zwischen Primat und Kollegialität. Das Verhältnis von Papst und Bischöfen auf dem Ersten und Zweiten Vatikanischen Konzil, in: Zentralismus statt Kollegialität? Kirche im Spannungsfeld, hrsg. von F. König, Düsseldorf 1990, pp. 82-113, qui p. 105.

${ }^{46}$ Cfr. soprattutto LG 26,1; CD 11,1 e 6,3. Sul significato costituzionale della formula in quibus et ex quibus cfr. W. Aymans, Die Communio Ecclesiarum als Gestaltgesetz der einen Kirche, AfkKR 139(1970), pp. 69-90.

${ }^{47}$ Per un ulteriore approfondimento di questi tre significati della formula conciliare cfr. E. Conecco, Taufe, in: R. Ahlers - L. Gerosa - L. Müller, Ecclesia a Sacramentis. 
Questo principio dell'immanenza reciproca, emergente in modo paradigmatico a livello della struttura costituzionale della Chiesa ma informante anche tutte le altre dimensioni della realtà ecclesiale, in particolare quella antropologica del fedele cristiano ${ }^{48}$, è conoscibile nella sua essenza solo attraverso la fede. Si verifica quello che Medard Kehl ha giustamente osservato nel suo libro Wohin geht die Kirche? («Dove va la Chiesa?»): «Anche come mistero la Chiesa resta sempre - tanto come causa finale della volontà salvifica universale di Dio quanto come grandezza realizzata nella storia - unità nella molteplicità e non unità prima della molteplicità. Questa originaria e dialettica "reciproca intima compenetrazione" di unità e molteplicità, più concretamente di Chiesa universale e Chiese particolari, la Chiesa la deve ultimamente alla sua origine teologico-trinitaria, dal momento che in Dio l'unità della natura divina è realizzata appunto solo nella trinità delle persone (e non prima di esse) e solo in questo modo si fa efficace all'interno della creazione. Per questo nel giorno di Pentecoste, a Gerusalemme, la Chiesa appare allo stesso tempo come Chiesa universale e come concreta Chiesa particolare» ${ }^{49}$.

Per questa ragione anche e soprattutto nel considerare la dimensione canonistica della communio Ecclesiarum è necessaria un'impostazione metodologica corretta, onde evitare di riprodurre a livello giuridico le riduzioni filosofiche e politiche spesso già consumate a livello ecclesiologico.

Theologische Erwägungen zum Sakramentenrecht, Paderborn 1992, pp. 27-36; ID., Ius universale-Ius particulare, in: AA.VV., Ius in vita et in missione Ecclesiae. Acta Symposii internationalis Iuris Canonici occurrente $\mathrm{X}$ anniversario promulgationis CIC (19-24.4.1993 in Civitate Vaticana), Città del Vaticano 1994, pp. 553-574, qui citato da: E. Corecco, Ius et communio. Scritti di Diritto Canonico, cit., Vol. I, pp. 549-573, soprattutto pp. 551-552.

${ }^{48}$ Cfr. E. Corecco, Erwägungen zum Problem der Grundrechte des Christen in Kirche und Gesellschaft. Methodologische Aspekte, AfkKR 150(1981), pp. 421-453, ora anche in: ID., Ordinatio fidei, op. cit., pp. 161-189.

${ }^{49}$ Cfr. M. KeHL, Wohin geht die Kirche, op. cit., pp. 92-93. Sull'origine teologico-trinitaria della Chiesa come Communio, si veda anche P. J. Cordes, Communio. Utopie oder Programm, Freiburg-Basel-Wien, pp. 147-156. 
Si tratta cioè di evitare contemporaneamente sia la tentazione latente nella tradizione ortodossa, che tende a concepire la Chiesa universale secondo il principio platonico degli universalia ante res ${ }^{50}$, per cui essa è un modello o archetipo trascendente che non esiste concretamente in se stesso ma si realizza solo nelle singole Chiese particolari ed in modo sempre uguale; sia quella latente nella tradizione protestante, che tende a concepire la Chiesa universale secondo il principio nominalista universalia post res ${ }^{51}$, per cui essa non esiste se non come risultato di una decisione volontaristica, ossia come federazione delle Chiese particolari, le sole ad esistere veramente. Si tratta pure di evitare una banalizzazione politica di queste opposte tendenze, oggi presenti nella Chiesa cattolica, applicandovi necessariamente etichette come quella della centralizzazione e decentralizzazione del potere ${ }^{52}$.

${ }^{50}$ Anche il documento Communionis notio della Congregazione per la Dottrina della Fede (Lettera a tutti i vescovi su alcuni aspetti della Chiesa come Communio, del 28 maggio 1992) sembra in un certo senso presupporre l'accettazione di questo principio; cfr. a questo proposito E. CoRECCO, Ius universale-ius particulare, op. cit., p. 557; L. Müller, Das Recht im Leben und in der Sendung der Kirche. Erwägungen zum internationalen kanonistischen Symposion vom 19. bis zum 24. April 1993 im Vatikan, AfkKR 162(1993), pp. 608- 634, qui pp. 631-634. Per una critica più dettagliata del documento cfr. M. KeHL, Wohin geht die Kirche, op. cit., pp. 89-98. Quest'ultimo fa comunque presente che nel commento pubblicato dal Cardinale Joseph Ratzinger sull'Osservatore Romano del 23.6.1993, dal tenore di una vera e propria retractatio, si riconosce come la reciproca immanenza (natura interioritas, Nr. 9) fra Chiesa universale e Chiesa particolare sia il concetto ermeneutico chiave di tutto il documento Communionis notio (cfr. M. KeHL, Wohin geht die Kirche, op. cit., p. 95).

${ }^{51} \mathrm{Su}$ come la preminenza ontologica attribuita da una certa ecclesiologia contemporanea alla Chiesa particolare faccia capo a questo principio nominalista $\mathrm{cfr}$. E. Corecco, Ius universale-ius particulare, op. cit., p. 558 e G. Colombo, La teologia della Chiesa locale, in: AA.VV., La Chiesa locale, a cura di A. Tessarolo, Bologna 1970, pp. 17-38.

${ }^{52}$ Altrettanto sbagliata, e per lo meno inadeguata sotto il profilo metodologico, è da considerare l'applicazione meccanica in ecclesiologia del principio della sussidiarietà, uno dei capisaldi dell'insegnamento sociale della Chiesa, dato che la Chiesa non è riducibile ad una semplice società umana; a tale riguardo cfr. 
In altri termini, come l'unicità di Dio è conoscibile solo attraverso la trinità delle persone, analogamente l'universalità della Chiesa può realizzarsi solo attraverso la pluralità delle singole Chiese. «La Chiesa di Cristo - ha affermato con vigore Eugenio Corecco - non potrebbe mai coincidere con un unico vescovo ed un'unica diocesi, fosse pure quella di Roma, perché verrebbe a mancare l'elemento essenziale della pluralità, che è il presupposto della comunione» ${ }^{53}$.

Ciò significa che a livello strettamente strutturale, e quindi del diritto costituzionale canonico, Chiesa universale e Chiesa particolare non sono due realtà materiali diverse e separabili: la formula conciliare dell'in quibus et ex quibus impone - come ha affermato esplicitamente nel 1984, ossia trent'anni orsono, Giovanni Paolo II proprio a Lugano, la città sede dell'Istituto di diritto canonico e diritto comparato delle religioni, che chi vi parla rappresenta - di considerarle come due dimensioni formali di un'unica realtà: la Chiesa di Cristo $^{54}$. Certo sul piano strettamente salvifico quest'ultima, ossia la «Ecclesia Christi» che nel simbolo professiamo come «unam, sanctam, catholicam et apostolicam» (LG 8,2) e come tale «subsistit in Ecclesia catholica» (LG 8,2), gode della priorità ontologica sua qualsiasi forma di inveramento strutturale o istituzionale.

«Infatti - come chiarisce e conferma la ormai famosa lettera "Communionis notio", pubblicata il 28 maggio 1992 dalla Congregazione per la dottrina della fede - "ontologicamente la Chiesa-Mistero", la Chiesa una ed unica secondo i padri addirittura precede la creazione e partorisce le chiese particolari come figlie, si esprime in esse, è

E. Corecco, Dalla sussidiarietà alla comunione, Communio 127(1993), pp. 90105, ora anche in: ID., Ius et Communio, op. cit., pp. 531-548; J. BeYer, Principe de subsidiarité ou «juste autonomie» dans l'Eglise, NRT 108(1986), pp. 801-822; F. X. KaUfMANn, The principle of subsidiarity viewed by the sociology of organisations, The Jurist 48(1988), pp. 275-291.

${ }^{53}$ E. Conecco, Ius universale-ius particulare, op. cit., p. 557.

${ }^{54}$ Cfr. Giovanni Paolo II, Omelia, Lugano 12 giugno 1984, in: Insegnamenti di Giovanni Paolo II, Città del Vaticano 1984, Vol. VII/1, pp. 1676-1683, qui nn. 3-4. 
madre e non prodotto delle Chiese particolari» ${ }^{55}$. Tuttavia questa priorità ontologica sul piano salvifico non può essere in alcun modo ridotta, a livello strutturale del diritto costituzionale canonico della Chiesa Cattolica, né all'identificazione della Chiesa Universale con la Chiesa di Roma, né tanto meno a principio fondamentale di rinnovata promozione del "centralismo romano". Lo evidenzia nel 1988 lo stesso Papa Giovanni Paolo II con la pubblicazione del Motu proprio "Apostolos suos" sulla natura teologica e giuridica delle Conferenze dei Vescovi ${ }^{56}$. Anzi, a onor del vero, un'altra delle grandi novità e diversità del pontificato di Giovanni Paolo II sta proprio nell'aver rilanciato, anche e soprattutto con i suoi viaggi apostolici, i legami tra la Santa Sede e le Chiese locali, ponendo chiaramente un freno al cosiddetto "centralismo romano" 57 .

E ciò a partire da Puebla, perché se è vero che nel complesso l'immagine ecclesiologica emersa in questa grande Assemblea sinodale sudamericana non è sempre univoca e del tutto priva di qualche confusione terminologica, dall'altra è altrettanto vero che la presenza del Papa Giovanni Paolo II ha avuto come risultato più incisivo quello di imprimere alla tematica stessa una forte prospettiva missionaria ${ }^{58}$, a tutto vantaggio sia del dibattito squisitamente teorico, sistematico ed ecclesiologico, sia di quello più pratico-pastorale.

Se a livello ecclesiologico e quindi più teorico, il principio della contemporanea originarietà (=Gleichursprünglichkeit), indispensabile per cogliere il risvolto strutturale dell'immanenza reciproca e totale dell'universale nel particolare conoscibile solo per fede, impedisce soprattutto di attribuire una priorità storica ed ontologica al particolare

\footnotetext{
${ }^{55}$ Congregazione Per la Dottrina Della Fede, Lettera "Communionis notio", AAS 85(1993), pp. 838-850 n. 9,9, qui citata da: Enchiridion Vaticanum, vol. 13, pp. 926-953, p. 937.

${ }^{56}$ Il testo ufficiale si trova in: AAS 90(1998), pp. 641-658.

${ }^{57}$ Cfr. S. Dziwisz, Ho vissuto con un santo, op. cit., p. 53.

${ }^{58}$ A tale riguardo cfr. E. Conecco, Prospettiva per la "Lex Ecclesiae fundamentalis" e la revisione del diritto canonico nel documento di Puebla, Il diritto ecclesiastico 1(1980), pp. 3-23, qui pp. 6-7.
} 
sull'universale, a livello pastorale e giuridico esige una equipollente valutazione delle categorie di integrazione o diversificazione.

L'applicazione giuridico-pastorale corretta di queste categorie implica il riconoscere, che da una parte la Chiesa universale è pienamente Chiesa solo se le Chiese particolari non sono uniformate, ma manifestano un'effettiva pluralità, dall'altra queste ultime a loro volta sono veramente Chiesa solo se concepiscono la loro particolarità all'interno dell'universalità della communio Ecclesiarum. Affinché ciò avvenga è necessario non solo il riconoscimento da parte del Papa o del Collegio dei vescovi della plena communio ${ }^{59}$, ma anche e forse soprattutto che Santa Sede e Papa sappiano sempre valorizzare l'apporto specifico delle Chiese particolari alla realizzazione della communio Ecclesiarum. Come ha affermato Eugenio Corecco nel suo lucido commento canonistico ai documenti di Puebla, se sotto il profilo rigorosamente teorico ed ecclesiologico è indiscutibile che i "due poli fondamentali" della costituzione ecclesiale della communio Ecclesiarum sono e rimarranno sempre da una parte le Diocesi, o Chiese particolari per antonomasia, e dall'altra la Chiesa Universale, per cui e di conseguenza i cosiddetti corpi ecclesiali intermedi (ossia i patriarcati, le province ecclesiastiche, le regioni rette da conferenze episcopali, ecc.) sono semplicemente delle "realtà derivate", sotto il profilo missionario e pastorale proprio queste realtà giuridiche, alcune antichissime ed altre di recentissima istituzione ${ }^{60}$, possono rappresentare una grande opportunità di sviluppo armonico ed organico del diritto particolare canonico, imprescindibile per una effettiva realizzazione della "Communio Ecclesiarum"61.

\footnotetext{
${ }^{59}$ Tale riconoscimento non crea però la comunione, ma semplicemente ne constata l'esistenza; cfr. in merito: E. CoRecco, Ius universale-ius particolare, op. cit., p. 556. ${ }^{60}$ Fra quelle di recente istituzione vanno ricordati i cosiddetti ordinariati personali; sulle differenze e complementarietà fra queste strutture pastorali personali e le Chiese particolari o locali, cfr. A. CATtAneo, Le strutture pastorali personali: unità e pluralità nella comunione ecclesiale, in: Veritas et Jus (autunno 2014), in corso di pubblicazione.

${ }^{61}$ Cfr. E. Conecco, Prospettiva per la "Lex Ecclesiae fundamentalis" e la revisione del diritto canonico nel documento di Puebla, op.cit., p. 6 e soprattutto p. 11.
} 
Ora cosa significa tutto ciò a livello del diritto costituzionale canonico nonché del significato concreto della norma generale del can. 20 ? E e soprattutto a livello dei rapporti tra diritto universale e diritto particolare, cosi come li ha intesi Giovanni Paolo II?

\subsection{Le conseguenze della communio Ecclesiarum a livello dei} rapporti diritto universale-diritto particolare

Prima di ogni considerazione specifica va subito affermato a chiare lettere che nella Chiesa diritto e comunione non sono due entità opposte, bensì elementi coevi ed inseparabili della sua struttura Costituzionale. Infatti, dato che la specifica socialità ecclesiale non nasce da un dinamismo naturale ma dalla grazia (Parola di Dio, Sacramento e Carisma), da una parte la communio rappresenta la realtà strutturale ed antropologica in cui si incarna la grazia con la sua forza ultimamente vincolante, dall'altra il diritto canonico non ha come suo fine ultimo semplicemente quello di garantire la realizzazione della giustizia, bensì la realizzazione della communio Ecclesiae et Ecclesiarum.

Quest'ultima rappresenta perciò lo statuto ontologico del diritto della Chiesa e come tale il principio formale di tutto il diritto canonico. Di conseguenza anche il principio dell'immanenza reciproca fra universale e particolare, che definisce l'essenza stessa della comunione a livello strutturale, informa a tal punto il sistema giuridico della Chiesa che in esso non può esserci - in linea di principio - alcuna opposizione concorrenziale fra l'aspetto universale e quello particolare.

a) Il «corpus iuris commune» della Chiesa di Cristo e i criteri di differenziazione fra diritto universale e diritto particolare

Dal Concilio di Trento al Vaticano II, se nell'ecclesiologia la tradizione latina ha privilegiato la Chiesa universale, nella canonistica, e soprattutto a livello delle codificazioni del diritto della Chiesa, il metodo giuridico l'ha fatta talmente da padrone su quello teologico da far prevalere quasi sempre il diritto universale su quello particolare.

Tuttavia oggi la formula conciliare dell'in quibus et ex quibus impone di riconoscere, nell'insieme di tutte le norme canoniche, l'esistenza di un corpus che appartiene all'unica Chiesa di Cristo, e 
come tale comune sia al Codex iuris Canonici che al Codex Canonum Ecclesiarum orientalium. A questo corpus, che potrebbe essere designato come corpus iuris commune ${ }^{62}$, appartengono quali suoi elementi costitutivi tutte le norme di diritto divino e quelle di diritto umano comuni a tutte le Chiese particolari ${ }^{63}$. In quanto tali queste norme canoniche non appartengono in modo specifico né alla Chiesa universale né a quelle particolari, bensì all'unica Chiesa di Cristo. Nella misura in cui esprimono dei contenuti della Chiesa di Cristo, e quindi qualcuno di quei suoi elementi costitutivi in cui si attua la salvezza comunicata dalla Parola di Dio e dai Sacramenti, è ultimamente irrilevante il fatto che esse siano state formulate dal Papa o dai Vescovi. Decisivo risulta invece il loro essere patrimonio comune della communio Ecclesiarum, per cui a rigore potrebbero essere considerate alla stessa stregua delle norme canoniche formulate da un Concilio ecumenico, «nel quale l'immanenza della Chiesa universale in quella particolare, grazie alla presenza del ministero petrino e di tutti i vescovi in rappresentanza delle loro Chiese particolari, si esprime in modo perfetto» ${ }^{64}$.

Il fatto che tutte le norme canoniche esplicitanti un contenuto del diritto divino positivo appartengono a questo corpus iuris commune è evidente: esse non sono norme specifiche né della Chiesa universale, né di quella particolare perché Parola e Sacramenti sono elementi costitutivi della Chiesa di Cristo in quanto tale, e perciò appartengono in modo uguale sia alla Chiesa universale sia alle Chiese particolari.

Per quanto riguarda le norme canoniche di diritto umano o mere ecclesiasticum, che sono diventate nel corso dei secoli patrimonio comune della communio Ecclesiarum, va invece osservato che

\footnotetext{
${ }^{62}$ Anche se E. Corecco (cfr. Ius universale-Ius particulare, op. cit., pp. 564-565) con uno sguardo al CIC parla di corpus commune legum, qui si tratta però più in generale di un corpus iuris e non solo di leggi in senso stretto; cfr. a tale riguardo M. Werneke, Ius universale-Ius particulare. Zum Verhältnis von Universal-und Partikularrecht, op. cit., p. 92.

${ }^{63}$ Cfr. E. Corecco, Ius universale-Ius particulare, op. cit., pp. 559-566; M. WerNEKe, Zum Verhältnis von Universalund Partikularrecht, op. cit., pp. 91-100.

${ }^{64}$ E. Conecco, Ius universale-Ius particulare, op. cit., p. 564.
} 
esse appartengono allo stesso corpus iuris commune solo in forza dell'istituto della ricezione o receptio, operante nei due sensi opposti, dal particolare all'universale e viceversa, come è ampliamente dimostrato dalla storia della Chiesa e soprattutto dalla storia dei suoi concili di ogni ordine. La forza vincolante di queste norme - normalmente applicabili solo ai fedeli cattolici ${ }^{65}$ - va comunque valutata alla luce dei due principi seguenti, comuni a tutta la tradizione canonistica: 1) Il diritto umano per definitionem rimane sempre riformabile; 2) Qualsiasi «legge umana, che contraddice la legge divina e naturale, è priva di validità» («lex humana, legi divinae et naturali contraria irrita est» ${ }^{66}$.

Queste brevi osservazioni generali sull'esistenza di un corpus iuris commune dovrebbero bastare, nonostante le difficoltà insite nella sua definizione quantitativa e qualitativa, a relativizzare il problema della preminenza del diritto universale su quello particolare e viceversa. All'interno di questo corpo comune è infatti impossibile una differenziazione concettuale fra gli elementi universali e quelli particolari.

Solo al di fuori di esso tale distinzione è possibile, anche se per nulla facile e nemmeno sempre in grado di stabilire dei confini netti fra i due settori. Essa potrebbe essere comunque così formulata: «Il diritto universale comprende tutte quelle norme (primarie o secondarie,

${ }^{65}$ Cfr. can. 11 CIC/1983; can. 1490/CCEO.

${ }^{66}$ Cfr. D. 8 c. 1; cfr. anche STh I-II, q. 95 a. 2 ad 4, q. 96 a. 4 . Lo ius canonicum in tutto il suo sviluppo sistematico ha sempre la sua ultima fonte effettiva nel diritto divino, da cui procede non solo la sua esistenza, ma anche il suo carattere giuridico. Di conseguenza, sarebbe sbagliato vedere la totalità delle norme canoniche di diritto divino positivo come assolutamente immutabili o la totalità delle norme canoniche di diritto esclusivamente ecclesiale come riformabili a proprio arbitrio. Mentre il primo insieme può essere definito come un diritto rivelato, che attraverso la tradizione è riconoscibile o reso visibile, il secondo, sebbene non sia una concretizzazione diretta del dato biblico, resta strettamente legato al processo di concretizzazione storico-normativa del diritto divino positivo o naturale. La distinzione classica tra ius divinum e ius mere ecclesiasticum è pertanto da intendere semplicemente come un tentativo di operare una prima distinzione fondamentale tra i contenuti oggettivi del diritto canonico, per determinare poi con più precisione la forza vincolante di ogni singola norma. Cfr. in proposito Aymans - Mörsdorf, KanR I, pp. 32-37. 
scritte o non scritte) che valgono per tutta la Chiesa, indipendentemente dal fatto che per loro natura obblighino tutti i fedeli o solo alcune categorie di persone... Come diritto particolare può essere pertanto designato quel diritto che non vale per tutta la Chiesa, sia a motivo di una limitazione territoriale o personale, oppure a causa di una limitazione sia territoriale che personale» ${ }^{67}$.

b) Funzione specifica e problemi di fondo del processo legislativo universale e particolare

Le funzioni specifiche del diritto universale e di quello particolare vanno definite a partire dal loro punto di riferimento comune, cioè il corpus iuris commune ${ }^{68}$, e perciò non possono che essere equivalenti, dovendo entrambi garantire - sia pure in modo diverso - il realizzarsi ed il permanere dell'essenza stessa della communio Ecclesiarum, secondo il principio dell'in quibus et ex quibus. Mentre il diritto universale deve garantire l'unità, senza ridurla ad uniformità, il diritto particolare deve invece garantire la pluralità senza cadere nel particolarismo o settarismo.

Nel corso dei secoli, la tradizione canonica ha saputo sviluppare non pochi istituti giuridici atti a regolamentare ed armonizzare questo duplice movimento. Per difendere l'identità delle Chiese particolari di fronte a scorretti interventi legislativi di tipo centralistico o centralizzatore sono stati ad esempio sviluppati istituti canonici come il cosiddetto ius remostrandi dei vescovi diocesani, oppure quello

\footnotetext{
${ }^{67}$ M. WERNEKE, Ius universale-Ius particulare. Zum Verhältnis von Universal-und Partikularrecht, op. cit., pp. 102-103. Sulla distinzione fra leggi universali e leggi particolari cfr. pure cann. 12.13.20 del CIC; cann. 1491.1502 del CCEO e il commento di P. Krämer, Kirchenrecht II. Ortskirche-Gesamtkirche, Stuttgart- Berlin-Köln 1993, pp. 57-62, soprattutto p. 59.

${ }^{68}$ Come questo riferimento al centro comune sia importante per salvare la specificità del diritto della Chiesa in ogni suo settore è percepito anche dalla teologia protestante del diritto canonico; cfr. a questo proposito R. DreIER, Der Rechtsbegriff des Kirchenrechts in juristisch-rechtshistorischer Sicht, in: AA.VV., Das Recht der Kirche, Vol. I: Zur Theorie des Kirchenrechts, hrsg. von G. Rau - H.R. Reuter - K. Schlaich, Gütersloh 1997, pp. 194-195.
} 
dell'effettiva non receptio legis o ancora quello della tacita desuetudo da parte del Popolo di $\mathrm{Dio}^{69}$. In questa luce va pure interpretata la norma generale del can. 20 .

Per togliere invece ogni legittimità ad iniziative legislative di tipo particolaristico si è rigorosamente fissato al can. $135 \$ 2$, sempre delle norme generali, il principio secondo cui «a legislatore inferiore lex iuri superiori contraria valide ferri nequit» ${ }^{70}$.

c) L'importanza missionaria e pastorale delle cosiddette strutture intermedie ed in particolare della provincia ecclesiastica

Nell'ecclesiologia del Concilio Vaticano Il è fuori di dubbio che solo il vescovo diocesano, in forza della pienezza del sacramento dell'ordine, è in possesso di tutta la sacra potestas ${ }^{71}$. È altresì fuori di dubbio che questo elemento personale, dovuto all'origine sacramentale della sacra potestas, deve sempre coniugarsi con l'elemento sinodale dell'esercizio di tale potestà, perché i due elementi sono reciprocamente immanenti l'uno nell'altro nella Chiesa come comunione. Da ciò deriva, ed è forse questo ciò che fa particolarmente fatica ad essere recepito in Svizzera, che la maggior partecipazione e corresponsabilità nell'esercizio del potere ecclesiale non si ottiene con l'ormai mitica "democratizzazione" della struttura costituzionale della Chiesa, che per diritto divino è ad un tempo gerarchica e carismatica (LG 4), ma con uno sviluppo articolato degli strumenti

${ }^{69}$ Cfr. R. PuzA, Katholisches Kirchenrecht, Heidelberg 1993, pp. 20-22; W. Schulz, Konsens und Widerspruch als rechtserhebliches Handeln in der Kirche? - Kanonistische Erwägungen zur Rezeptionsproblematik, ThGl 81(1991), pp. 339-351; J. B. HARING, Das bischöfliche Vorstellungsrecht gegenüber dem Apostolischen Stuhl, AfkKR 91(1911), pp. 111-114.

${ }^{70}$ Can. $135 \$ 2$. Sugli antecedenti storici di questo principio generale della legislazione canonica, cfr. P. Hinschius, System des Katholischen Kirchenrechts. Mit besonderer Rücksicht auf Deutschland, III, Berlin 1883, pp. 829-832; G. PHILLIPS, Kirchenrecht, III/1, Regensburg 1848, p. 667.

${ }^{71}$ Sull'argomento cfr. L. Gerosa, Der Bischof: Seine Bestellung, seine geistliche Vollmacht und die christliche Verkündigung in Europa, Bulletin-ET 3(1992), pp. 66-94. 
e della mentalità sinodale propri alla communio Eclesiarum $^{72}$, che presuppone sempre anche la corresponsabilità di tutti i fedeli cattolici, come ha chiaramente evidenziato l'Assemblea di Puebla ${ }^{73}$.

All'interno di questo insegnamento del Concilio Vaticano II, di grande importanza per tutto il diritto canonico, si comprende più facilmente come i padri del Concilio in LG 23, 4 e CD 40 abbiano voluto rivalutare il ruolo pastorale delle province ecclesiastiche non solo per ragioni storiche, ma anche soprattutto per ragioni ecclesiologiche dettate dalla nuova autocoscienza della Chiesa come comunione di Chiese.

Il Codice di diritto canonico del 1983 nei cc. 431-459 ha recepito la sostanza di questa lezione conciliare: infatti il c. $431 \S 2$ prescrive che di regola non ci possono più essere diocesi esenti e che ognuna di esse dovrebbe essere perciò integrata in una provincia ecclesiastica, che possiede di per sé - a differenza del raggruppamento di Chiese particolari sul territorio di una Conferenza episcopale nazionale - la personalità giuridica (c. $432 \$ 2$ ). Gli organi di governo di una provincia ecclesiastica sono il Metropolita ed il Concilio provinciale. Mentre il primo organo di governo è legato ad una sede episcopale, il secondo non solo ha potestà di governo ma soprattutto possiede per diritto, a differenza delle Conferenze episcopali, potestà legislativa (c. 445), per tutte le Chiese particolari raggruppate nella stessa provincia (c. 440 \$1). La struttura sinodale della provincia ecclesiastica ha dunque una base giuridica solida, sia pure sotto alcuni aspetti ancora perfezionabile ${ }^{74}$. Tuttavia a livello pastorale è difficile contraddire il giudizio di chi ritiene che le norme codiciali ad essa relative siano purtroppo

\footnotetext{
${ }^{72}$ Per un'analisi dettagliata della questione cfr. L. Gerosa, Rechtstheologische Grundlagen der Synodalität in der Kirche Einleitende Erwägungen, in: W. Aymans K. T. GeHringer (a cura di), Iuri canonico promovendo. Festschrift für H. Schmitz zum 65 Geburtstag, Regensburg 1994, pp. 35-55.

${ }^{73}$ Cfr. E. Conecco, Prospettiva per la "Lex Ecclesiae fundamentalis" e la revisione del diritto canonico nel documento di Puebla, op.cit., p. 13.

${ }^{74}$ Sarebbe ad esempio auspicabile un ulteriore miglioramento del profilo canonistico del Concilio provinciale, soprattutto per ciò che concerne la scelta degli oggetti da trattare. Alla stessa stregua dovrebbe essere preso in considerazione un maggior
} 
rimaste finora lettera morta ${ }^{75}$. Non si conosce infatti il tentativo di un metropolita di convocare un Concilio provinciale, per cui è più che legittimo chiedersi perché mai questa reticenza, proprio ora che alle mega-conferenze dei vescovi viene rimproverato di favorire una certa messa in ombra della responsabilità personale di ogni singolo vescovo diocesano. Molto probabilmente le attuali polarizzazioni ideologiche intra-ecclesiali e l'ostinazione con cui si continua a parlare di collegialità, anche quando il termine ecclesiologicamente e canonisticamente più appropriato è quello di sinodalità, hanno in qualche modo impedito a teologi e vescovi di constatare come il profilo codiciale della provincia ecclesiastica dimostri ampiamente che la stessa è un'istituzione canonica, in cui l'elemento personale e quello sinodale dell'esercizio della sacra potestas si integrino armoniosamente. Se si tiene inoltre presente che la sinodalità presuppone sempre per sua natura la corresponsabilità dei laici, allora si capisce che senza far ricorso a riduzioni democratiche, teologicamente illegittime, dell'esperienza ecclesiale, sia possibile concludere che la provincia ecclesiastica possa essere considerata uno strumento di grande aiuto nell'organizzazione futura della missione e della pastorale ${ }^{76}$. E i motivi sono almeno tre.

In primo luogo nella definizione giuridica della provincia ecclesiastica, che non è di diritto divino bensì di mero diritto umano, il criterio del territorio gioca un ruolo più importante che non nella definizione della Chiesa particolare, concepita ormai in senso più personalistico. Ciò le conferisce una capacità maggiore in ordine all'inculturazione della fede, senza tuttavia scadere in riduzioni di tipo nazionalistico, perché il territorio di una provincia ecclesiastica di regola non corrisponde mai a quello di una nazione.

coinvolgimento dei vescovi suffraganei, dei Capitoli cattedrali e altri gremi nella procedura di designazione del metropolita.

${ }^{75}$ Cfr. H. Maritz, Die Stellung des Metropoliten im neuen kirchlichen Recht, in: A. Gabriels - H. J. F. Reinhardt (a cura di), 'Ministerium iustitiae'. Festschrift für H. Heinemann, Essen 1985, pp. 243-247, qui p. 246.

${ }^{76}$ In merito, cfr. L. Gerosa, Il principio conciliare della Communio Ecclesiae et Ecclesiarum e il problema della nuova divisione delle diocesi svizzere, Revista Espanola di Teologia 62(2012), pp. 253-264. 
In secondo luogo la provincia ecclesiastica pur possedendo una marcata struttura sinodale a differenza della regione ecclesiastica, governata collegialmente da una Conferenza episcopale ${ }^{77}$, è presieduta da un metropolita. In essa non solo l'elemento sinodale si coniuga armonicamente con quello personale, ma proprio in forza di questa reciproca immanenza ai suoi organi di governo, il diritto canonico riconosce competenze maggiori. Ad esempio la provincia ecclesiastica a norma del c. $377 \$ 3$ - assume infatti un rilievo non indifferente nel procedimento di nomina dei vescovi delle diocesi appartenenti alla propria circoscrizione: a norma del c. 1438 al tribunale metropolitano spettano inoltre i diritti di tribunale d'appello ordinario ${ }^{78}$. Il già citato Concilio provinciale è poi un organo di governo di ben altro peso che una semplice Conferenza episcopale regionale. Infatti, da una parte a norma del c. $443 \$ 3$ anche le seguenti persone sono membri ordinari - sia pure solo con voto consultivo - di un Concilio provinciale: i vicari generali ed episcopali, i superiori maggiori degli istituti di vita consacrata, i rettori delle Università ecclesiastiche e dei Seminari, i delegati del Capitolo cattedrale e dei Consigli presbiterale e pastorale; dall'altra la funzione del Concilio provinciale è eminentemente legislativa (praesertim legislativa, c. 445) e i suoi decreti necessitano solo della recognitio e non della approbatio da parte della Santa Sede per essere promulgati (c. 446).

In terzo ed ultimo luogo non va dimenticato che per la piena rivalutazione dell'ufficio di metropolita e del ruolo pastorale della provincia ecclesiastica in futuro potrebbe risultare estremamente importante il c. $436 \$ 2$ che recita: «Dove le circostanze lo richiedono, la Sede Apostolica può conferire al metropolita funzioni e potestà peculiari da determinare nel diritto particolare». Ed il ruolo del diritto particolare e del pluralismo giuridico all'interno della Chiesa cattolica come communio Ecclesiarum è stato ampiamente dimostrato da Giovanni Paolo II sia con la promulgazione del Codice per le Chiese cattoliche orientali (CCEO) sia con la convocazione, di diversi sinodi continentali

\footnotetext{
${ }^{77}$ Cfr. CD 11 e c. 369.

${ }^{78}$ Cfr. c. 434.
} 
o regionali. Inoltre, sempre a questo proposito basta ricordare che già il Concilio di Trento accordava ai metropoliti la possibilità non solo di supplire iure devolutivo la negligenza dei suffraganei ma anche di erigere, in caso di necessità, dei Seminari interdiocesani o provinciali. E non c'è ragione alcuna di dubitare che queste possibilità potranno essere ulteriormente ampliate, visto che anche il c. 381 del Codice di diritto canonico del 1983 riconosce al vescovo diocesano tutte le competenze necessarie all'esercizio del suo ufficio pastorale ad eccezione di quelle che di diritto o per decreto pontificio vengono riservate ad altra autorità ecclesiastica.

\section{Conclusione}

Quando il 2 giugno 2005 Suor Marie Simon-Pierre, gravemente ammalata del morbo di Parkinson e poi guarita miracolosamente durante il processo di beatificazione di Karol Wojtyla, si presenta dalla sua superiora per chiederle di esonerarla da ogni attività lavorativa, si sente rispondere "Giovanni Paolo II non ha ancora detto la sua ultima parola"79. Ebbene questa frase lapidaria può essere metaforicamente applicata anche a Giovanni Paolo II quale grande legislatore e riformatore del diritto canonico. Infatti, le due parti centrali di questa conferenza possono essere viste come il dittico centrale di un polittico comprendente diversi altri pannelli non ancora dipinti. Il loro abbozzo o disegno iniziale è però già contenuto nei due pannelli centrali, quello relativo alle basi della nuova ermeneutica canonistica e quello relativo alla non concorrenzialità fra diritto universale e diritto particolare della Chiesa Cattolica. Entrambi irradiano una forza innovativa ed una ricchezza di prospettive di ulteriori sviluppi davvero assolutamente straordinarie. Occorre però che il canonista contemporaneo sappia coglierle e svilupparle senza paure e preconcetti al servizio della comunione e della missione della Chiesa. Per fare questo il suo cuore e la sua mente devono innanzitutto lasciarsi aprire alla categoria del "dono". Si, perché anche a livello del diritto

\footnotetext{
${ }^{79}$ A. Tornielli, L'ultimo miracolo, op. cit., p. 120.
} 
canonico «l'eredità di Giovanni Paolo II è un dono»" ${ }^{80}$ ! Una simile affermazione conclusiva non può e non deve sorprendere o addirittura irretire il canonista contemporaneo, perché essa definisce compiutamente anche il grande sforzo innovativo e missionario compiuto da San Raimondo di Penyafort all'inizio del II millennio cristiano e che gli permise di essere più tardi riconosciuto universalmente come santo patrono dei canonisti ${ }^{81}$. Infatti, il suo grazie a Papa Gregorio IX, espresso nel 1234 all'atto della consegna delle "Decretali" da lui redatte, è di una tale densità che può benissimo essere assunto come motto programmatico da ogni canonista contemporaneo che -confrontato con le sfide culturali di questo inizio del III millennio cristiano- desidera con umiltà e determinazione far propria la grande eredità canonistica di San Giovanni Paolo II, riaffermando nei suoi confronti: Quod dedisti nobis custodivimus!

\section{Universal e particular law in the light of the canonical hermeneutic of St. John Paul II}

While the mystical expression "to see beyond" allows John Paul II to "trace the direction" for the development of a new canonical hermeneutic, apt for assuming the great missionary challenges of the of the third millennium, the normative novelty introduced by him and the methodological direction dealt by him allow his conviction, that the Church is "a house and school of communion", to become the programmatic force of all the structural reforms initiated by him. This reform of the Church, which is communio Ecclesiarum, in its communion and missionary aspects, finds its central core at the level of the relationships between the universal law and the particular law. Or rather, it is precisely the so called corpus iuris commune that, thanks to John Paul II's methodological and hermeneutic direction, can redefine the specific and interactive functions existing between universal canon law and particular canon law: the first must guarantee the unity,

\footnotetext{
${ }^{80}$ S. Dziwisz, Ho vissuto con un santo, op. cit., p. 145.

${ }^{81}$ In merito, cfr. F. Valls - I Taberner, San Raimondo di Penyafort. Padre del diritto canonico, Bologna 1998, pp. 78-86.
} 
without reducing it to uniformity: the second instead should guarantee the plurality, without falling into particularism.

PARole-Chiave: Giovanni Paolo, diritto universale e particolare, ermeneutica canonistica, communio Ecclesiarum.

KEY wORDS: John Paul II, universal and particular law, canonical hermeneutic, communio Ecclesiarum.

\section{Nota o Autorze:}

Prof. Libero Gerosa - Nel 1984 consegue il Dottorato in Teologia all'Università di Friburgo e nel 1988 ottiene la libera docenza in Diritto canonico all'Università Cattolica di Eichstätt. Dal $1990 \mathrm{al} 2000$ è professore ordinario di Diritto canonico alla Facoltà di Teologia di Paderborn, dal 1997 al 1999 Rettore della stessa Facoltà e dal 2000 al 2008 Rettore della Facoltà di Teologia di Lugano. Nel 2002 fonda l'Istituto Istituto Internazionale di Diritto Canonico e Diritto Comparato delle Religioni presso la medesima Facoltà e ivi è stato professore ordinario di Diritto canonico fino al 2015, data del suo pensionamento. Ė attualmente docente di Ermeneutica biblica alla Facoltà di Teologia di Lugano. Dal 2001 è Consultore del Pontificio Consiglio per i Laici, e dal 2003 Consultore della Congregazione per il Clero. Nel 2008 è stato nominato Cappellano di Sua Santità e dal 2014 è Canonico onorario dell’arcidiocesi di Przemyśl. 